\title{
Structural Pounding Detection by Using Wavelet Scalogram
}

\author{
Shutao Xing, ${ }^{1}$ Marvin W. Halling, ${ }^{2}$ and Qingli Meng ${ }^{3}$ \\ ${ }^{1}$ DeepSea Engineering (US) Inc., Former at Utah State University, Houston, TX 77042, USA \\ ${ }^{2}$ Department of Civil \& Environmental Engineering, Utah State University, Logan, UT 84322, USA \\ ${ }^{3}$ Institute of Engineering Mechanics, China Earthquake Administration, Harbin 150080, China \\ Correspondence should be addressed to Shutao Xing, shutaoxing@yahoo.com
}

Received 19 June 2012; Accepted 26 August 2012

Academic Editor: K. M. Liew

Copyright (๑) 2012 Shutao Xing et al. This is an open access article distributed under the Creative Commons Attribution License, which permits unrestricted use, distribution, and reproduction in any medium, provided the original work is properly cited.

\begin{abstract}
Structural pounding can cause considerable damage and even lead to collapse of structures. Most research focuses on modeling, parameter investigation, and mitigation approaches. With the development of structural health monitoring, the on-line detection of pounding becomes possible. The detection of pounding can provide useful information of potential damage of structures. This paper proposed using wavelet scalograms of dynamic response to detect pounding and examined the feasibility of this method. Numerical investigations were performed on a pounding system that consisted of a damped single-degree-of-freedom (SDOF) structure and a rigid barrier. Hertz contact model was used to simulate pounding behavior. The responses and pounding forces of the system under harmonic and earthquake excitations were numerically solved. The wavelet scalograms of acceleration responses were used to identify poundings. It was found that the scalograms can indicate the occurrence of pounding and occurrence time very well. The severity of the poundings was also approximately estimated. Experimental studies were carried out, in which shake table tests were conducted on a bridge model that underwent pounding between its different components during ground motion excitation. The wavelet scalograms of the bridge responses indicated pounding occurrence quite well. Hence the conclusions from the numerical studies were verified experimentally.
\end{abstract}

\section{Introduction}

The different phase vibrations of neighboring buildings or adjacent parts of the same building or bridge can result in pounding under earthquake excitation if the separation distance between them is not sufficient. The pounding can cause considerable damage and even lead to collapse of structures, for example, the bridges with columns of unequal heights $[1,2]$. Damage of buildings and bridges due to pounding has been documented in the reports of many earthquakes by researchers. The existed pounding research concentrated on modeling of pounding systems, parameter investigations, and mitigation approaches.

Most mathematical pounding modeling methods fall into two categories: (a) stereomechanical impact approach, which is primarily based on impulse-momentum law; (b) contact element approach, a force-based approach. The first approach was used in [3] and other literature. Hertz contact model, a contact element approach, has been extensively used to model impact [4-7]. The numerical study portion of this paper used this model.

With the development of structural health monitoring systems in bridges and other civil structures, the online detection of the presence of pounding and parameter determination of the pounding system becomes possible. Valuable information about pounding can be extracted from the response measurements to provide early warning about damage to the structures. There is rare research on this useful topic based on the authors' knowledge. Wavelets allow for powerful and flexible methods for dealing with fundamental engineering problems and have been used within a broader scope [8]. This paper proposed to use wavelet scalograms to detect the occurrence of pounding.

Wavelet scalogram is square of the modulus of the wavelet transform. It can uncover previously concealed information about the nature of nonstationary processes. It has been widely used in vibration signal analysis, which includes denoising, fault diagnostics, structural performance 


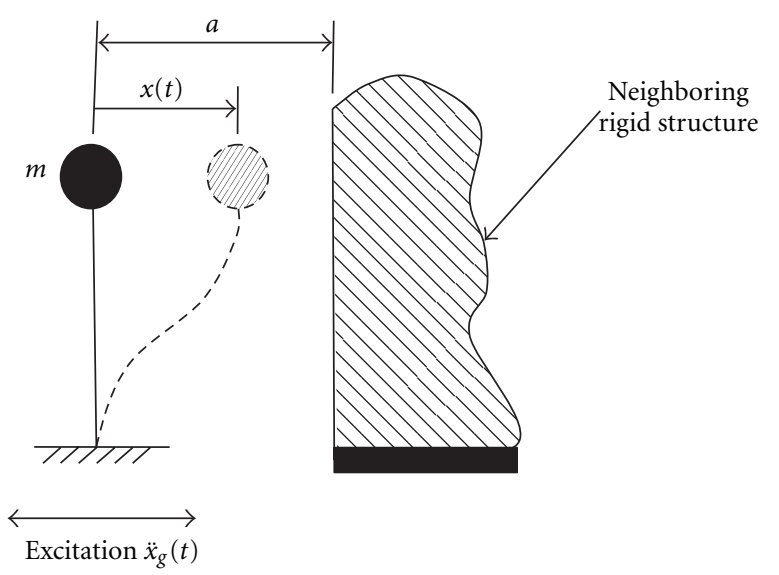

FIGURE 1: The impact oscillator for one-sided pounding.

testing, ground motion analysis, bridge response due to vortex shedding, and many other forms of analysis $[9,10]$.

The primary objective of this paper is to investigate the applicability of wavelet scalograms in seismic pounding detection. Numerical and experimental studies were conducted to achieve this aim. In the numerical study, the Hertz contact model was introduced into the pounding modeling, and the properties of a large-scale bridge segment were used for an SDOF concrete structure while the appropriate value of an impact stiffness parameter was calculated. The responses of the pounding system between the SDOF structure subjected to harmonic and earthquake excitations and a stationary rigid structure were computed and processed to obtain wavelet scalograms. In the experimental studies, shake table tests were conducted on a steel bridge model. The pounding between the different units of the bridge was detected by analyzing the dynamic response measurements. The effectiveness of the wavelet scalogram in identifying seismic pounding was studied.

\section{Investigation of the Pounding Model}

2.1. Pounding Model. In the numerical study, pounding was simulated by an impact oscillator, which was proposed by Davis [5] and later adopted in the literature $[4,6,7]$. An SDOF damped oscillator subjected to ground motion as illustrated in Figure 1 was studied. The pounding occurred between the oscillator and a neighboring stationary rigid structure. The neighboring structure can also be flexible structures; however, for the sake of simplicity, this paper investigated the rigid case only.

The equation of motion for the nonlinear pounding model of the SDOF system subjected to ground motion $\ddot{x}_{g}(t)$ in Figure 1 is

$$
m \ddot{x}(t)+c \dot{x}(t)+R(x, t)+F(t)=-m \ddot{x}_{g}(t),
$$

where $m$ denotes the system mass, $c$ denotes the damping coefficient, $\ddot{x}, \dot{x}, x$ denote the relative acceleration, velocity and displacement relative to the ground, the superimposed dot denotes differentiation with respect to time, and $R(x, t)$ is the restoring force.

This study focused on an elastic structure. For this case, the damping coefficient and restoring force can be expressed as

$$
c=2 \xi \sqrt{k m} ; \quad R(x, t)=k_{e} x(t),
$$

where $\xi$ and $k_{e}$ denote the damping ratio and initial stiffness, respectively.

$F(t)$ in (1) is the impact force between the colliding structures. Using the Hertz contact model, the impact force is represented by a nonlinear spring of stiffness $\beta$ and can be expressed as

$$
F(t)= \begin{cases}\beta[x(t)-a]^{3 / 2}, & \text { for } x(t)>a \\ 0, & \text { for } x(t) \leq a,\end{cases}
$$

where $a$ is the separation distance between the colliding structures. The Hertz exponent $3 / 2$ is a typical value to render (1) as nonlinear. While pounding in the real world may have different values, the alternative values have little effect on the system's response $[5,6] . \beta$ is the impact stiffness parameter, which is dependent on the material properties and geometric surfaces of the contact bodies [11]. The calculation of values of $\beta$ for some special cases can be found in [12]. For the pounding between concrete bodies, the values of $\beta$ typically range from $2 \sim 80 \mathrm{kN} / \mathrm{mm}^{3 / 2}$ as demonstrated in [11]. However, these values are based on small-scale experiments. They are not suitable for large-scale situations [7].

In the numerical examples used in this paper, the impact between a sphere of radius $R_{1}$ and massive plane surface of $R_{2}=\infty$ was considered. The appropriate impact stiffness parameter is calculated as

$$
\beta=\frac{4}{3 \pi} \sqrt{R_{1}} \frac{1}{\delta_{1}+\delta_{2}},
$$

where $\delta_{1}=\left(1-v_{1}^{2}\right) / E_{1} ; \delta_{2}=\left(1-v_{2}^{2}\right) / E_{2}, R_{1}$ is the radius of the flexible structure in Figure $1, E_{1}, E_{2}, \nu_{1}$, and $v_{2}$ are the elastic modulus and Poisson's ratios of the contact structures, respectively. For the neighboring rigid barrier, assuming $E_{2} \rightarrow \infty$, then $\delta_{2} \rightarrow \infty$, (4) becomes

$$
\beta=\frac{4}{3 \pi} \sqrt{R_{1}} \frac{E_{1}}{1-v_{1}^{2}}
$$

Equation (5) was used in Section 4 to calculate $\beta$.

2.2. Solution of the Equation of Motion. The equation of motion (1) was solved by using the Dormand-Prince 4thorder-runge-kutta method with adaptive size as well as the Dormand-Prince 8th-order-runge-kutta method. When the results of these two methods were compared, it was found that the 8th order method was no better than the former method. Therefore, only the results of the 4 th-order method were presented in this paper.

The solution method for the pounding system was verified by using a numerical model from [4]. The SDOF 


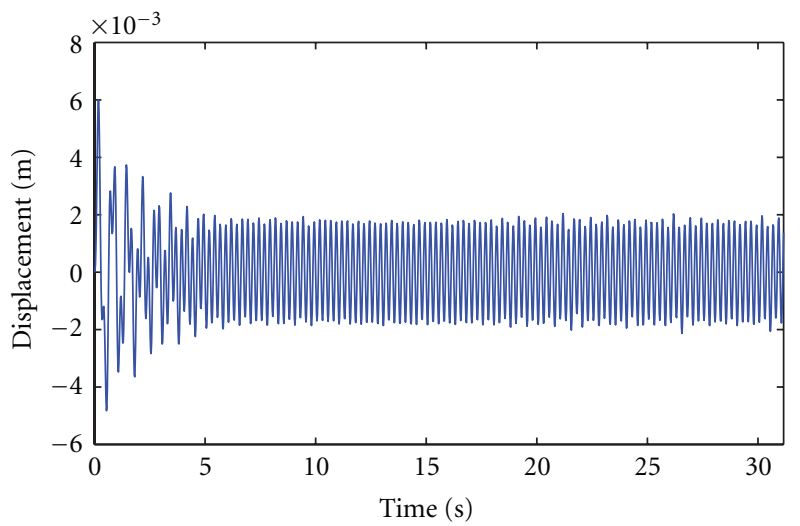

(a)

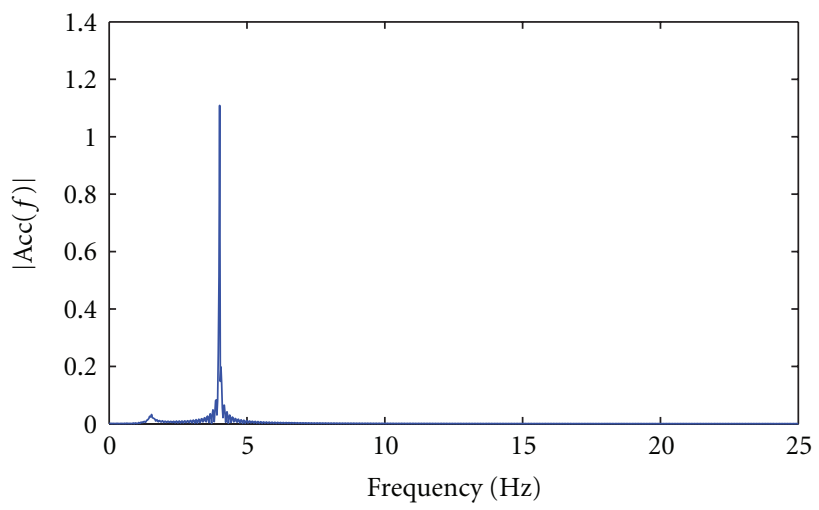

(c)

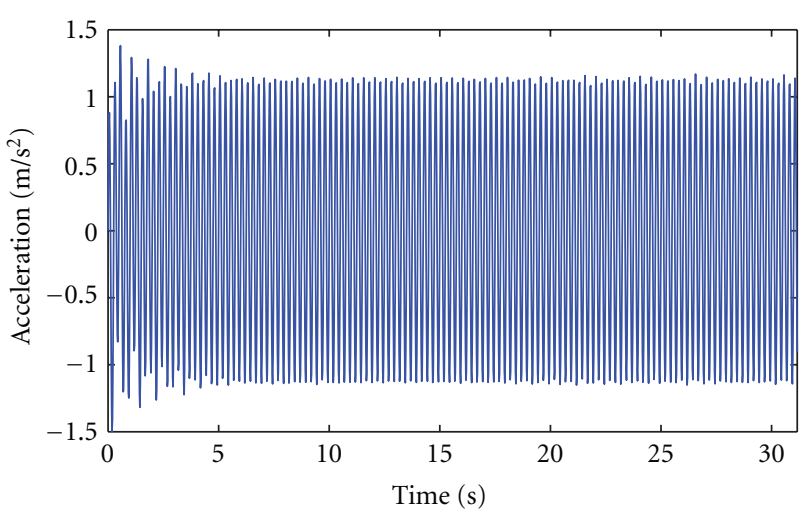

(b)

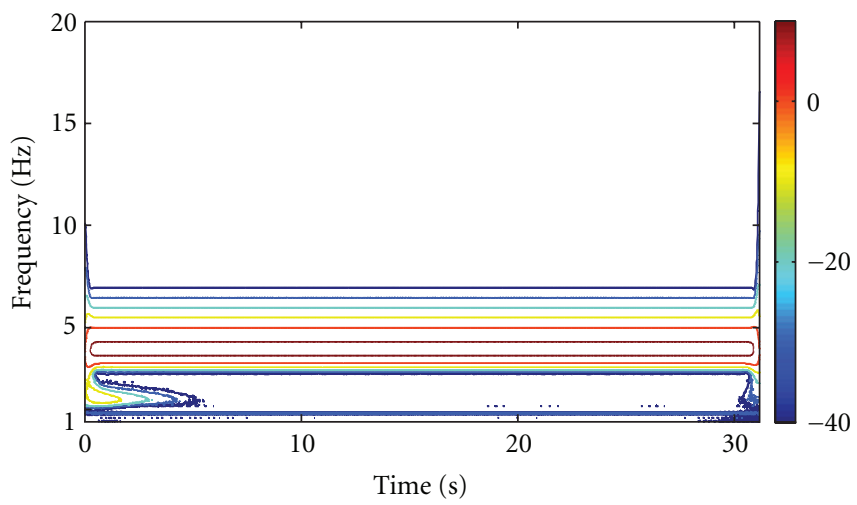

(d)

FIGURE 2: The responses of the SDOF structure during sinusoidal excitation $f_{1}=4 \mathrm{~Hz}$, no pounding occurred: (a) displacement; (b) acceleration; (c) single-sided amplitude spectrum of acceleration; (d) wavelet scalogram of acceleration response.

elastic system with mass $m=350 \mathrm{Mg}$, elastic stiffness $k_{e}=$ $10.5 \mathrm{MN} / \mathrm{m}$, damping ratio $\xi=0.02$, separation gap $a=$ $0.025 \mathrm{~m}$, and impact stiffness parameter $\beta=80 \mathrm{kN} / \mathrm{mm}^{3 / 2}$ was used as a verification example. Two cases of sine excitations with $2 \mathrm{HZ}$ and $0.67 \mathrm{HZ}$ sharing a common magnitude of $0.1 \mathrm{~g}$ were each applied separately. The responses of displacement, acceleration, and pounding force calculated in this study were compared against those presented in [4]. They matched perfectly, so the method in this paper was verified to be accurate. This comparison study was not presented herein.

\section{Wavelet Transform and Scalogram}

The wavelets $\psi_{a, b}(t)$ generated by dilation and translation from the analyzing wavelet $\psi(t)$ are defined as

$$
\psi_{a, b}(t)=|a|^{-1 / 2} \psi\left(\frac{t-b}{a}\right),
$$

where $a$ is a scale parameter and $b$ is a translation parameter.

$\psi(t)$ and $\psi_{a, b}(t)$ are usually called mother and son wavelets, respectively. It is supposed that $\psi(t) \in L^{2}(\mathbf{R})$ and the analyzing wavelet satisfy the admissibility condition

$$
C_{\psi}=\int|\omega|^{-1}|\widehat{\psi}(\omega)|^{2} d \omega<\infty,
$$

where $\hat{\psi}(\omega)=\int \psi(t) e^{-i \omega t} d t$ is the Fourier transform. It can be derived from the previous conditions that,

$$
\int \psi(t) d t=0 .
$$

Various wavelets have been developed, which include Haar, Morlet, Mexican Hat, Hermitian, and Daubechies wavelets. To select a proper mother wavelet is important. The Morlet wavelet is a complex function that can be considered as modulated Gaussian function by sinusoidal wave; it is a cosine signal decaying exponentially on both sides. It has the advantage of allowing trade between time and frequency resolution. It was proved to be an effective tool for feature extraction for mechanical vibration signals diagnosis [13, 14]. It has also been applied in seismic signal detection [15]. Morlet wavelet is similar to impulse component, which is symptom of damage in many dynamic signals. Because of these properties, the complex Morlet wavelet was chosen for this research, which is defined as

$$
\psi(x)=\frac{1}{\sqrt{\pi f_{b}}} e^{2 i \pi f_{c}^{x}} e^{-x^{2} / f_{b}},
$$

where $f_{b}$ is a bandwidth parameter and $f_{c}$ is a wavelet center frequency [16]. $f_{b}=1.5, f_{c}=1$ are used for the wavelet scalograms in Sections 4 and 5 of this paper. 


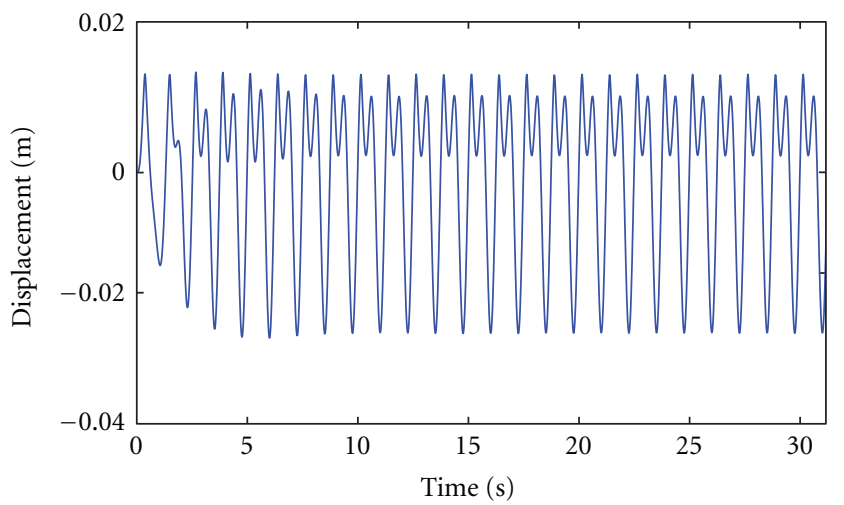

(a)

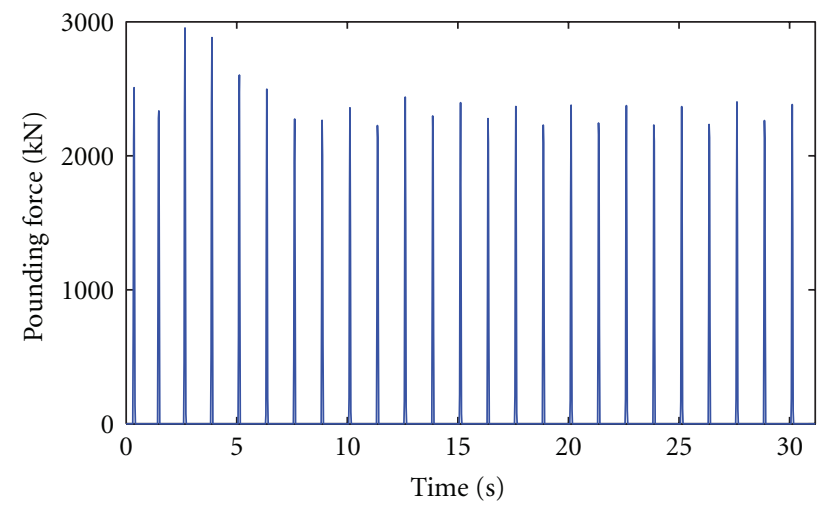

(c)

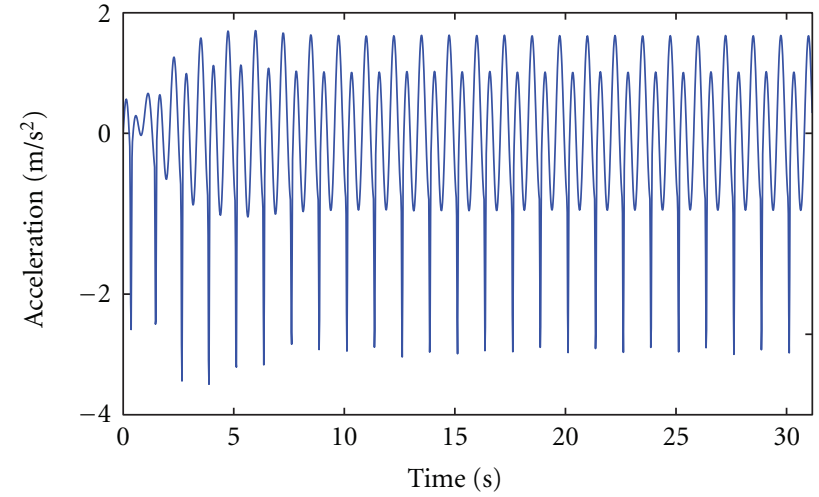

(b)

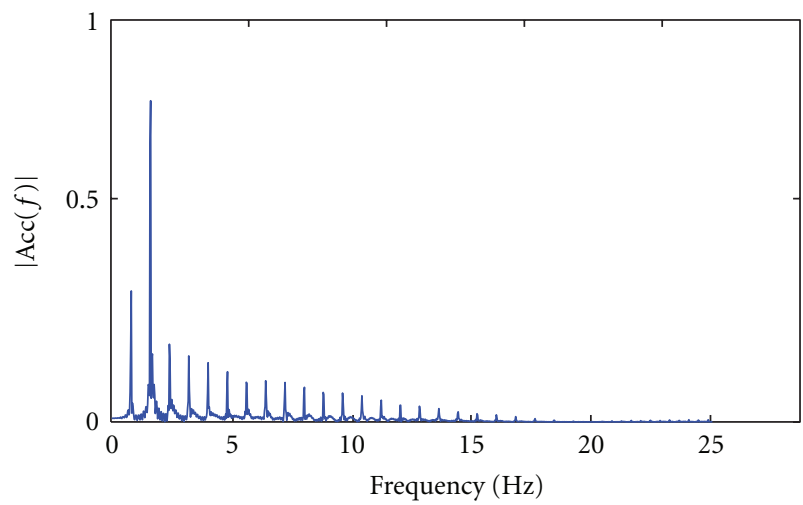

(d)

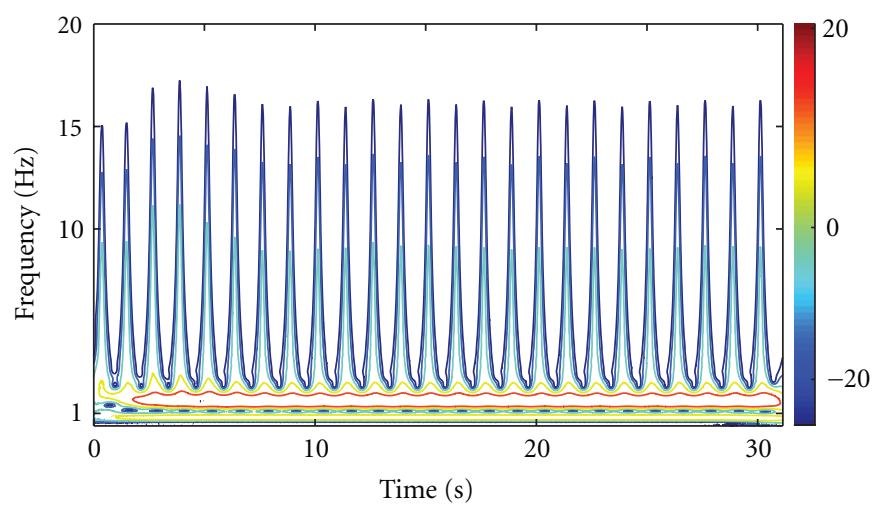

(e)

FIGURE 3: The responses of the SDOF structure during sinusoidal excitation $f_{2}=0.8 \mathrm{~Hz}$ : (a) displacement; (b) acceleration; (c) pounding force; (d) single-sided amplitude spectrum of acceleration; (e) wavelet scalogram of acceleration.

The wavelet transform is a linear transform that correlates a finite-energy function $x(t)$ with a series of oscillating functions $\psi_{a, b}(t)$ it is defined as

$$
W x(a, b)=|a|^{-1 / 2} \int x(t) \psi_{a, b}^{*}(t) d t>0
$$

where the asterisk denotes a complex conjugate. The modulus is defined as

$$
\|W x(a, b)\|=\left(\operatorname{Re}^{2}[W x(a, b)]+\operatorname{Im}^{2}[W x(a, b)]\right)^{1 / 2} .
$$

The phase is defined as

$$
\varphi(a, b)=\arctan \frac{\operatorname{lm}[W x(a, b)]}{\operatorname{Re}[W x(a, b)]} .
$$

The modulus and phase were used in [17] to investigate nonlinear and chaotic behavior of structural systems.

The wavelet scalogram is defined as the square of the modulus of the wavelet transform and represents energy 


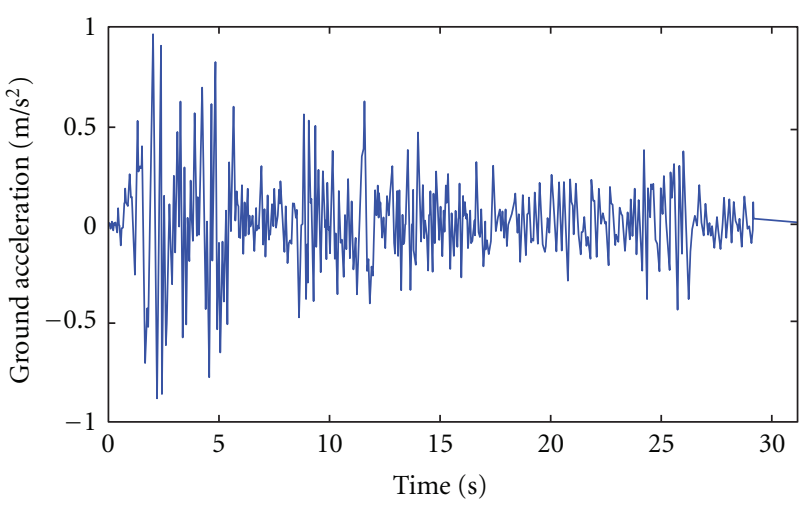

(a)

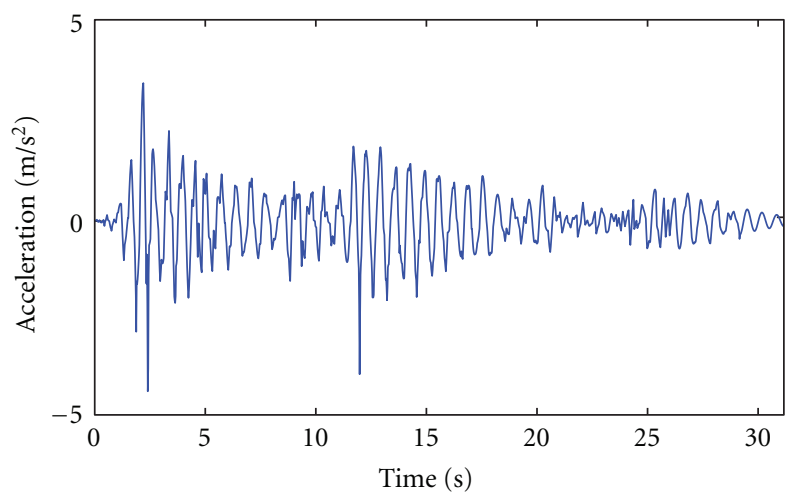

(c)

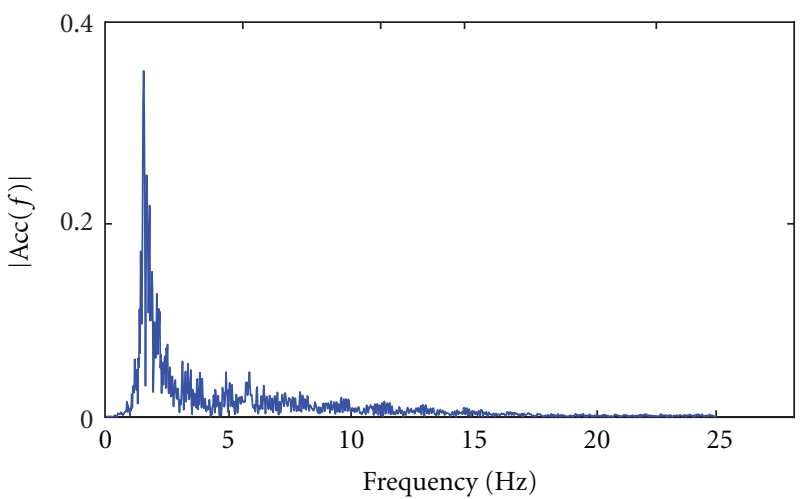

(e)

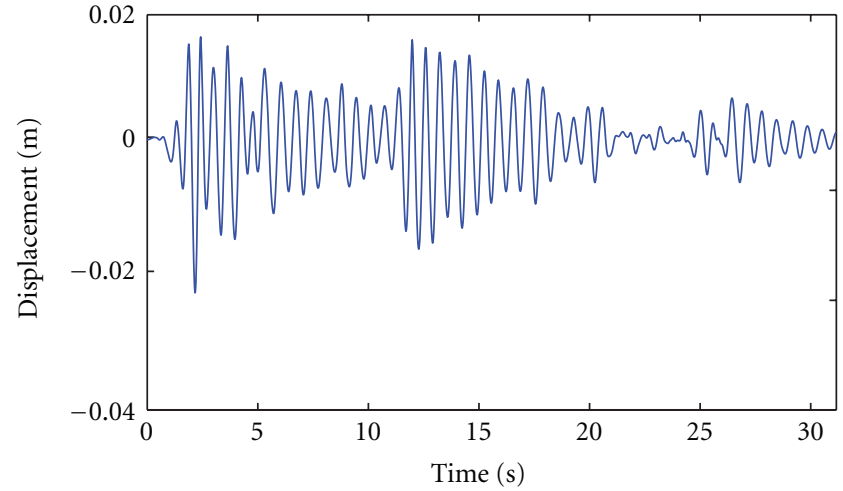

(b)

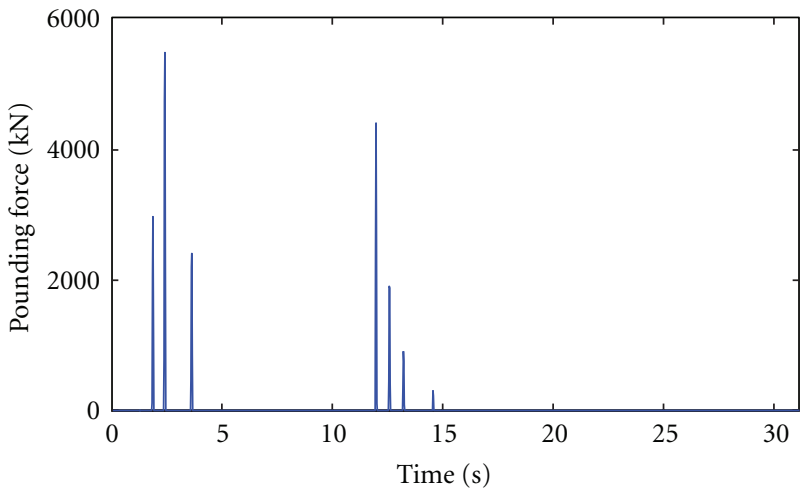

(d)

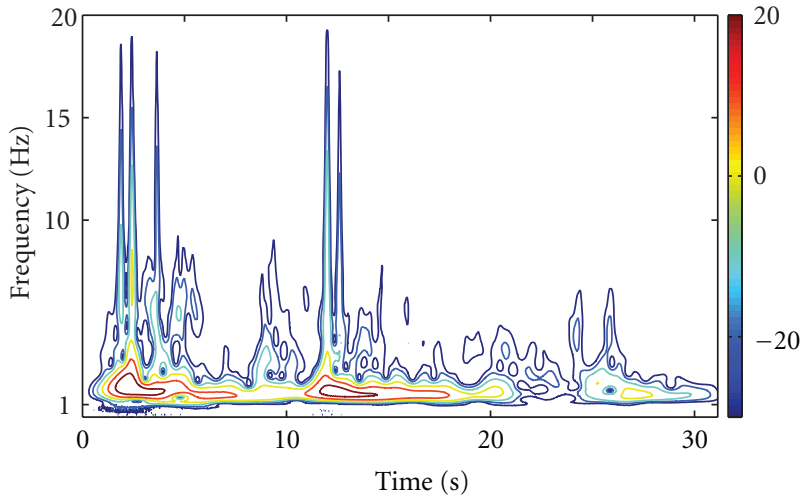

(f)

Figure 4: The El Centro earthquake excitation and responses of the SDOF structure: (a) ground acceleration, PGA = 0.1 g, (b) displacement response; (c) acceleration response; (d) pounding force; (e) single-sided amplitude spectrum of the acceleration; (f) wavelet scalogram of acceleration.

density that measures the energy of $x(t)$ in the Heisenberg box of each wavelet $\psi_{a, b}$ centered in [18]:

$$
P_{W} x(a, \xi)=\|W x(a, b)\|^{2}=\left\|W x\left(a, \frac{\eta}{\xi}\right)\right\|^{2} .
$$

It is used for analysis of nonstationary signals and can be understood as a spectrum with a constant relative bandwidth. The scalogram is employed in this study to determine the occurrence of pounding for both numerical and experimental data.

\section{Numerical Investigations}

The results of pounding of an impact oscillator on its neighboring rigid barrier, as illustrated in Figure 1, were investigated. The properties of the shorter concrete bridge segment in [3] were used for the SDOF structure: mass $m$ $=1.2 \times 10^{6} \mathrm{~kg}$, elastic stiffness $k_{e}=107 \mathrm{MN} / \mathrm{m}$, damping ratio $\xi=0.05$, and a natural period $T=0.67$ seconds. The other parameters were $R_{1}=4.92 \mathrm{~m}$ from [7], Poisson ratio $\nu_{1}$ $=0.15$, compressive strength $f_{c}^{\prime}=4000 \mathrm{psi}(27.58 \mathrm{MPa})$, and consequently concrete strength $E_{1}=57000 \sqrt{4000}=3.605 \times$ 


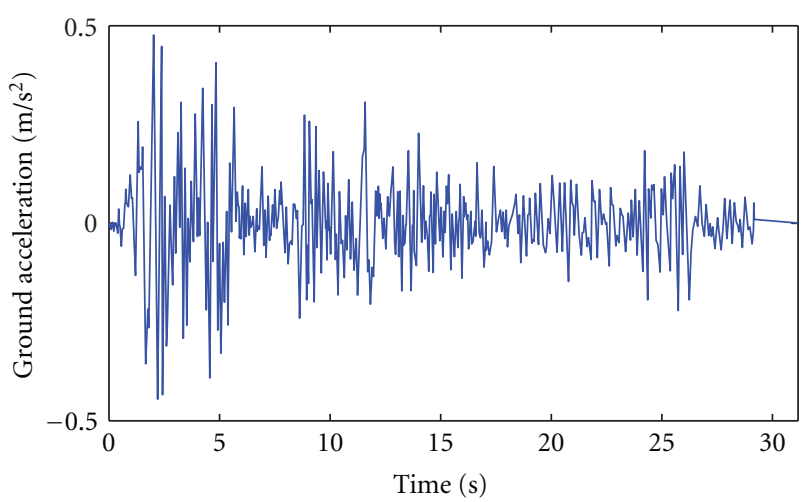

(a)

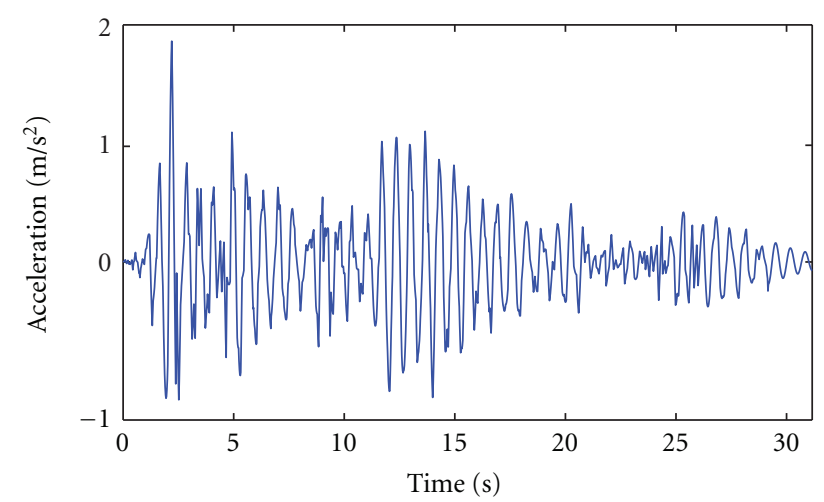

(c)

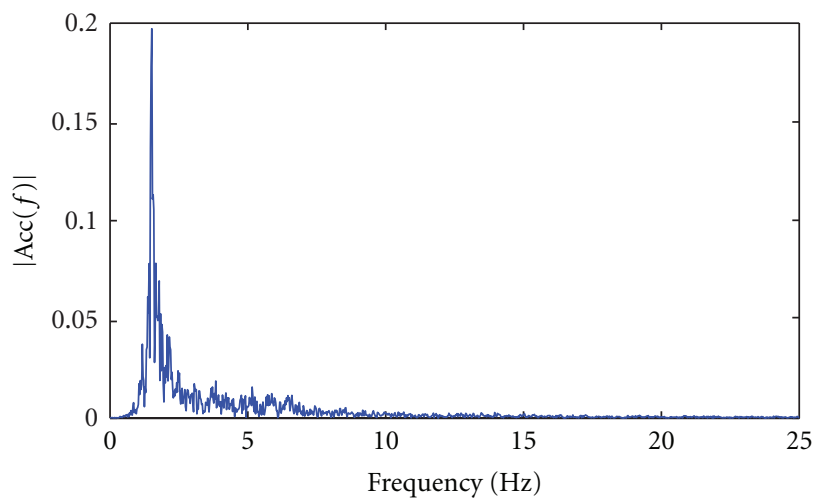

(e)

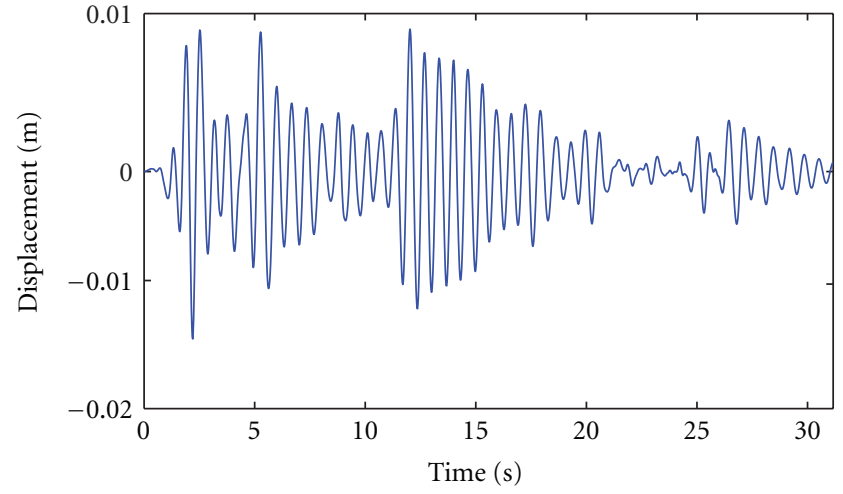

(b)

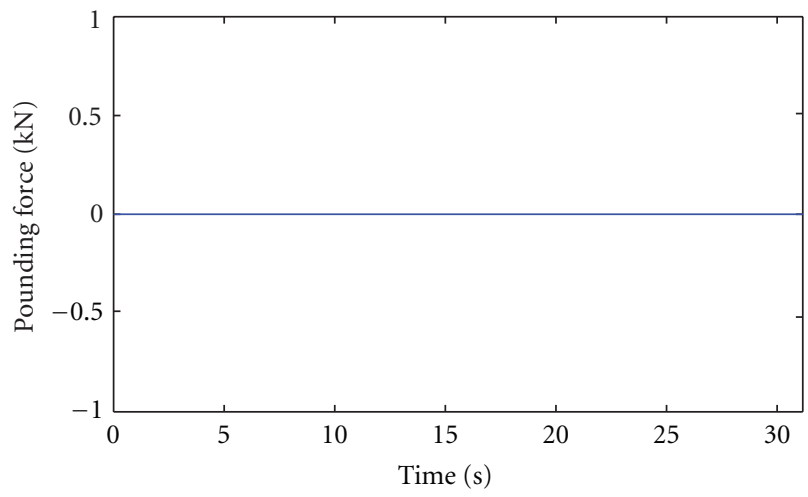

(d)

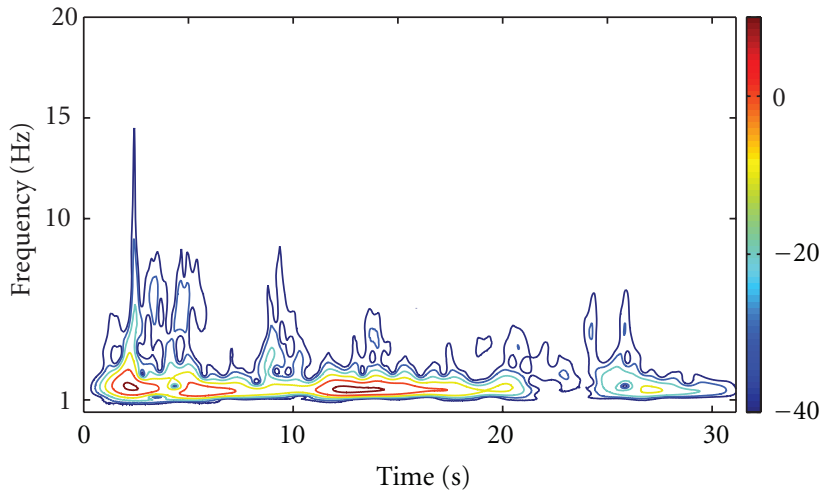

(f)

FIGURE 5: The El Centro earthquake excitation and responses of the SDOF structure: (a) ground acceleration, PGA = $0.05 \mathrm{~g}$, (b) displacement response; (c) acceleration response; (d) pounding force; (e) single-sided amplitude spectrum of the acceleration; (f) wavelet scalogram of acceleration.

$10^{6} \mathrm{psi}=24.856 \mathrm{GPa}$. Substitute the above values into (5) to calculate the impact stiffness parameter, and it was obtained that $\beta=757 \mathrm{kN} / \mathrm{mm}^{3 / 2}$. The separation distance was $a=$ $12.5 \mathrm{~mm}$. The structure was excited by two single frequency sinusoidal ground motions with frequencies $f_{1}=4 \mathrm{~Hz}$ and $f_{2}=0.8 \mathrm{~Hz}$, respectively: $\ddot{x}_{g}=-0.1 \mathrm{~g} \times \sin \left(2 \pi f_{1} t\right) ; \ddot{x}_{g}=$ $-0.1 \mathrm{~g} \times \sin \left(2 \pi f_{2} t\right) .1940$ El Centro North-South component scaled to peak ground motion (PGA) of $0.1 \mathrm{~g}$ and $0.05 \mathrm{~g}$, respectively, were also employed as ground excitations. The duration of 31.14 seconds was used for all the cases.

The responses of the pounding system were computed by the procedures discussed in Section 2. The wavelet scalograms of the acceleration responses were calculated by the method expanded upon in Section 3. The frequency axes of the scalograms were in log scale. The objective was to find a method to detect the presence of pounding from the time history signals that may include the displacement/velocity/acceleration. Both the amplitude spectra and scalogram were calculated from acceleration responses. The pounding forces that were computed numerically addressed the actual condition but were treated as unknown throughout the detection process.

The analyses of responses due to stationary sinusoidal excitation are discussed first. Figure 2 shows the responses, 


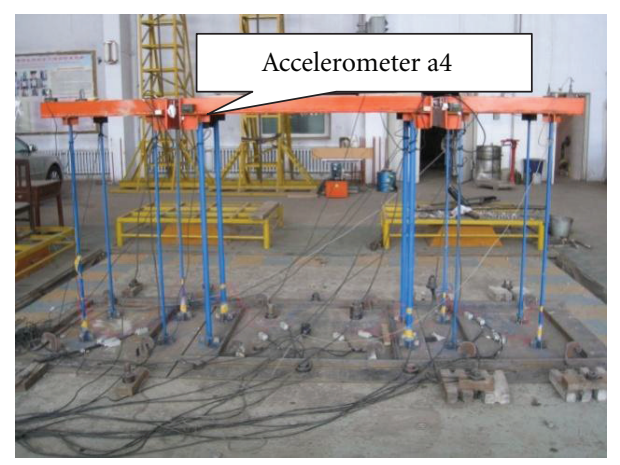

FIGURE 6: The pounding models of high-pier Bridge under shaking table tests by Meng et al. [19].

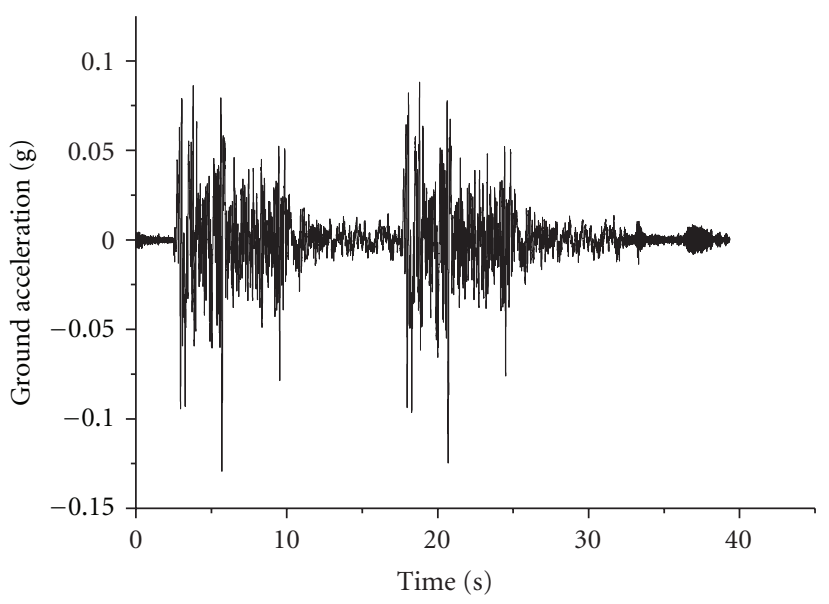

Figure 7: A typical table acceleration for the shake table tests.

single-sided amplitude spectra and scalogram for the case $f_{1}$ $=4 \mathrm{~Hz}$, at which pounding forces were all computed to zero during this session, thus indicating no pounding presence. The displacement and acceleration were able to maintain the approximately sinusoidal shapes after the first few seconds of oscillation. The single-sided amplitude spectrum shows that the predominant frequency is approximately same to the excitation frequency of $4 \mathrm{~Hz}$. The scalogram provdies a clear picture of energy distribution with respect to time and frequency.

Figure 3 shows the displacement, acceleration, pounding forces, single-sided amplitude spectra, and scalogram for the case $f_{1}=0.8 \mathrm{~Hz}$, which is closer to the natural frequency of the SDOF structure, and consequently generates larger displacement and acceleration responses. In Figures 3(a)$3(\mathrm{~b})$, the displacement and acceleration change to periodical signals after about 5 seconds. Although the responses were not regular as the nonpounding responses, there were no acceleration spikes and it was not certain whether pounding has occurred. The pounding forces as shown in Figure 3(c) indicate the exact time and duration of the poundings. The spectrum in Figure 3(d) shows many prominent frequencies, and additionally the predominant frequency is $1.6 \mathrm{~Hz}$ rather than the excitation frequency $0.8 \mathrm{~Hz}$, which indicates that pounding may have occurred at this time. But this judgment needs a priori knowledge of the structure and the excitation. Compared with the nonpounding scalogram shown in Figure 2(d), the scalogram in Figure 3(e) has many abrupt changes of energy with respect to the frequency. When Figures 3(e) and 3(c) are compared, it is obvious that these changes occur at the time of pounding. Further, the changes of frequency contents indicate the magnitude of the pounding force. Therefore the scalogram of acceleration response can indicate the presence of pounding, the time when pounding occurs, and relative intensity of the pounding.

The dynamic responses due to nonstationary earthquake excitation were analyzed. Figures 4 and 5 show the analysis results due to earthquake excitation with different PGAs. The earthquake record of 1940 El Centro northsouth component scaled to PGA of $0.1 \mathrm{~g}$ is illustrated in Figure 4(a), which contains its most energy approximately in the frequency range of $0.2 \sim 10 \mathrm{~Hz}$. It was observed that there are spikes in the acceleration histories shown in Figure 4(c), which indicates that the pounding may have occurred. The pounding forces shown in Figure 4(d) illustrate the presence of pounding due to this excitation. Unfortunately, the pounding cannot be detected by amplitude spectra very well. The wavelet scalogram for PGA $=0.1 \mathrm{~g}$ is illustrated in Figure 4(f), and the scalogram for PGA $=0.05 \mathrm{~g}$ that does not generate pounding is shown in Figure 5(f). When Figures $4(\mathrm{f})$ and $4(\mathrm{~d})$ are compared, it is clear that the higher frequencies determined by the scalogram are associated with the occurrence of pounding. The scalogram in Figure 5(f) for the nonpounding case does not show these types of changes, this additionally proves that the scalogram is capable of detecting pounding. It can be concluded that acceleration spikes and wavelet scalogram can detect pounding effectively.

\section{Experimental Data Analysis}

A series of shaking table tests were conducted on seismic pounding of a high-pier bridge by Meng et al. [19] at Institute of Engineering Mechanics, China Earthquake Administration in 2009. The measured accelerations were analyzed in this paper by using a wavelet scalogram to verify the ability of scalograms to detect pounding.

5.1. Shake Table Tests on Seismic Pounding of High-Pier Bridge Models. A high-pier bridge model with one main span and two approach spans was constructed as illustrated in Figure 6; the prototype of it was a high-pier bridge in Yunan, China. The superstructures and the $1.50 \mathrm{~m}$ tall piers were built with steel plates and pipes, respectively. The main span and approach spans had different sizes. Different ballast masses were added to satisfy the similitude laws. Consequently they had different natural frequencies. The gaps between the main span and the approach spans were $0.01 \mathrm{~m}$.

The bridge model was placed on the $5 \mathrm{~m} \times 5 \mathrm{~m}$ threedimensional shake table. Seven accelerometers, 11 displacement sensors, and 20 strain gauges were attached to the 


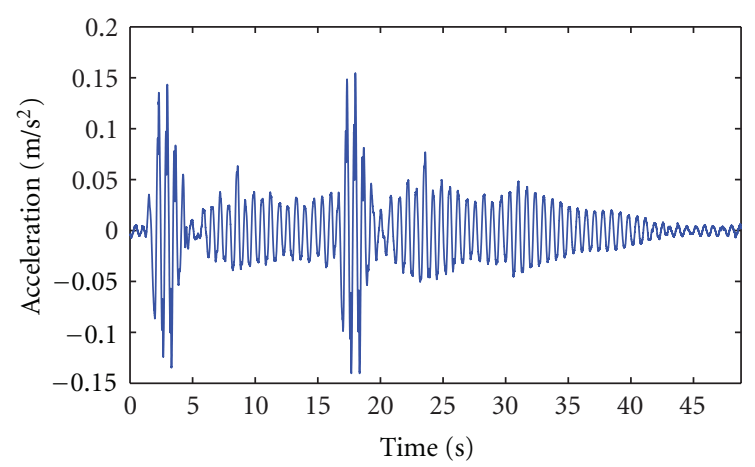

(a)

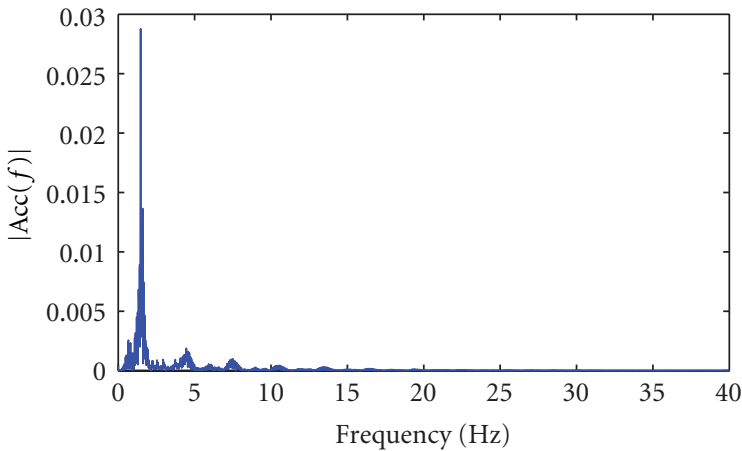

(b)

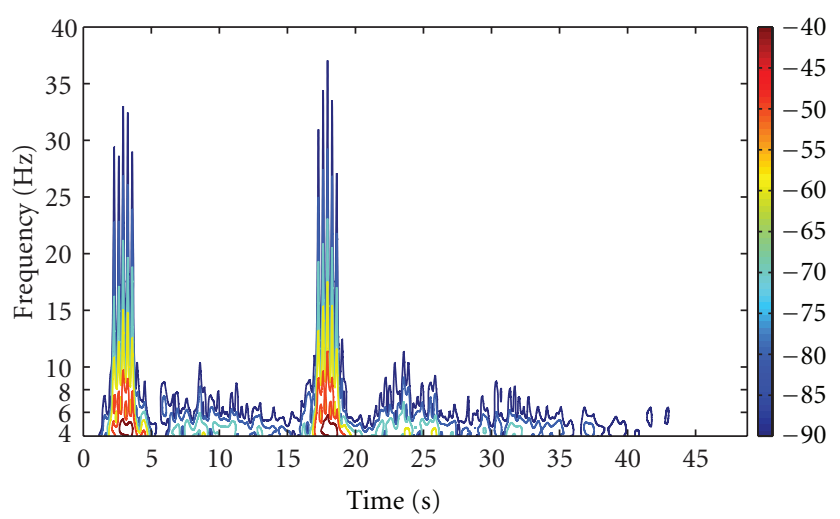

(c)

FIGURE 8: The response of the main bridge at Accelerometer a4 with SMAMRD: (a) acceleration response a4; (b) single-sided amplitude spectrum of the acceleration a4; (c) wavelet scalogram of a4.

bridge model. Two sets of dynamic tests were conducted on this model. The first tests were performed on the previous described bridge model, and the second tests were implemented on the previous model installed with shape memory alloy metal rubber dampers (SMAMRDs). The SMAMRD reduced the pounding effects significantly. The applied ground motions simulated the El Centro, Taft earthquake and artificial ground motions for both test sets with same PGAs. In this study, the accelerations a4 from the El Centro ground motion tests were analyzed. The PGA of the El Centro table motion was $0.22 \mathrm{~g}$ for both the test sets with and without SMAMRD. A typical table acceleration is shown in Figure 7.

5.2. Pounding Detection by Wavelet Scalogram. The accelerations recorded by accelerometer a4 for both test sets with and without SMAMRD were analyzed by wavelet scalograms. The results of the two tests are shown in Figures 8 and 9 respectively. The analysis methods were the same as those used in nummrical investigations.

From the acceleration response, single-sided amplitude spectra, and the scalogram in Figure 8 for the test set with SMAMRD, it is observed that in the time ranges around 3 and 18 seconds, the peak values of acceleration become 2 times as those of other time ranges and the scalograms extended to higher frequencies 3 times as those of other time ranges. The changes in the scalogram together with the acceleration indicate clearly that the pounding occurs at the time ranges around 3 and 18 seconds. However, no clear pounding information can be drawn from the spectra.

From the response results shown in Figure 9 for the test set without SMAMRD, it was observed that in the time ranges of 3.5 9.2, 15.0, 18.5 22, and 24.0 seconds, the peak values of acceleration become about 3 times as those of other time ranges and the scalograms extended to higher frequencies about 5 times those of other time ranges. Therefore, it can be concluded that the pounding occurs at the above time ranges by analyzing the acceleration spikes and wavelet scalograms. However, the pounding information from the spectrum is inconclusive.

Both test sets in Figures 8 and 9 refer to the same El Centro ground motion with the same PGA. Comparing the times of pounding and the scalogram that represent the energy density in Figures 8(c) and 9(c), the times of pounding occurrence and the scalogram magnitudes in Figure 9(c) are more frequent and larger than those of Figure 8(c). The scalogram in Figure 9 is extended to much higher frequencies of $100 \mathrm{~Hz}$ compared to about $37 \mathrm{~Hz}$ in Figure 8. These results are consistent with the conclusion in [19] that the SMAMRD can reduce pounding substantially. 


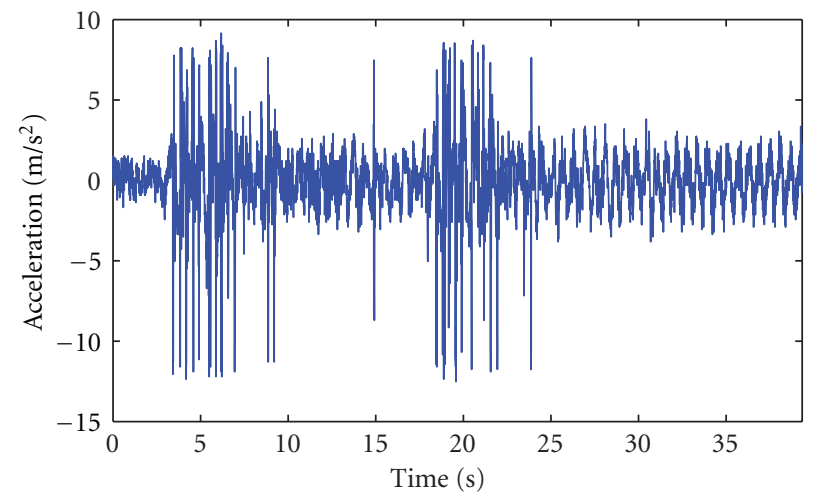

(a)

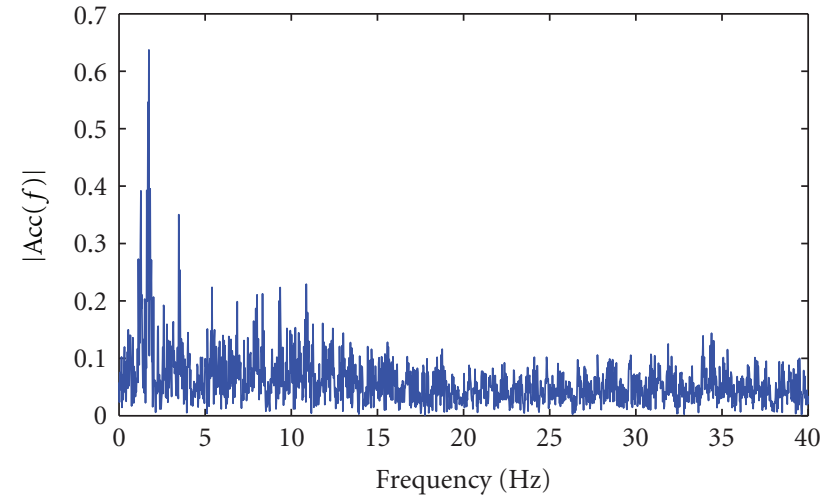

(b)

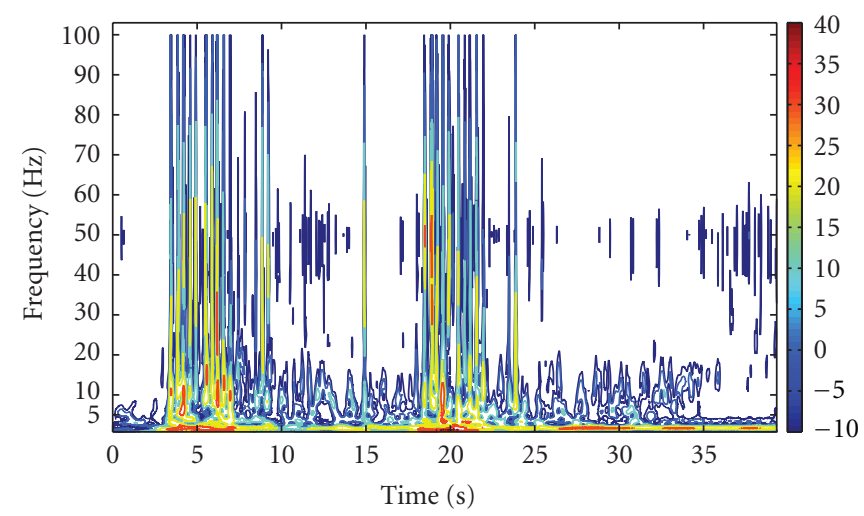

(c)

FIGURE 9: The response of the main bridge at Accelerometer a4 without SMAMRD: (a) acceleration response a4; (b) single-sided amplitude spectrum of the acceleration a4; (c) wavelet scalogram of a4.

\section{Conclusions}

In this paper, the pounding detection by wavelet scalogram was examined by numerical studies as well as experimental studies.

In the numerical studies, the Hertz contact model was carefully integrated into the one-sided pounding system between an elastic SDOF concrete structure and a rigid barrier. The pounding forces, displacement, and acceleration of the pounding system were solved from the numerical models for both stationary sinusoidal and non-stationary earthquake excitations. Acceleration spikes were present in the earthquake excitation case, but not in the harmonic one. The wavelet scalogram proved its ability to identify pounding occurrences in both cases. It can also be used to indicate the time of pounding occurrence and the approximate severity of each pounding session.

In the experimental studies, the acceleration data from two sets of shake table tests on a steel bridge model with and without SMAMRD were analyzed. Pounding occurred between the different components of the bridge for both sets of tests. The wavelet scalogram of the accelerations detected the pounding effectively, which verified the conclusions from the numerical studies. The acceleration spikes were also able to indicate the pounding for this case.
It can be concluded that the wavelet scalogram is capable of indicating the presence of structural pounding quite well. The acceleration spikes can be used together with the wavelet scalogram for pounding detection. Further analytical and experimental studies on more practical cases are needed.

\section{References}

[1] R. Jankowski, K. Wilde, and Y. Fujino, "Pounding of superstructure segments in isolated elevated bridge during earthquakes," Earthquake Engineering and Structural Dynamics, vol. 27, no. 5, pp. 487-502, 1998.

[2] M. J. N. Priestley, F. Seible, and G. M. Calvi, Seismic Design and Retrofit of Bridges, John Wiley and Sons, 1996.

[3] P. K. Malhotra, "Dynamics of seismic pounding at expansion joints of concrete bridges," Journal of Engineering Mechanics, vol. 124, no. 7, pp. 794-802, 1998.

[4] C. P. Pantelides and X. Ma, "Linear and nonlinear pounding of structural systems," Computers and Structures, vol. 66, no. 1, pp. 79-92, 1998.

[5] R. O. Davis, "Pounding of buildings modelled by an impact oscillator," Earthquake Engineering and Structural Dynamics, vol. 21, no. 3, pp. 253-274, 1992.

[6] K. T. Chau and X. X. Wei, "Pounding of structures modelled as non-linear impacts of two oscillators," Earthquake Engineering and Structural Dynamics, vol. 30, no. 5, pp. 633-651, 2001. 
[7] S. Muthukumar and R. DesRoches, "A Hertz contact model with non-linear damping for pounding simulation," Earthquake Engineering and Structural Dynamics, vol. 35, no. 7, pp. 811-828, 2006.

[8] J. S. Walker, A Primer on Wavelets and Their Scientific Applications, Chapman \& Hall/CRC, Boca Raton, Fla, USA, 1999.

[9] Z. K. Peng and F. L. Chu, "Application of the wavelet transform in machine condition monitoring and fault diagnostics: a review with bibliography," Mechanical Systems and Signal Processing, vol. 18, no. 2, pp. 199-221, 2004.

[10] K. Gurley and A. Kareem, "Applications of wavelet transforms in earthquake, wind and ocean engineering," Engineering Structures, vol. 21, no. 2, pp. 149-167, 1999.

[11] J. G. M. van Mier, A. F. Pruijssers, H. W. Reinhardt, and T. Monnier, "Load-time response of colliding concrete bodies," Journal of Structural Engineering, vol. 117, no. 2, pp. 354-374, 1991.

[12] W. Goldsmith, Impact: The Theory and Physical Behaviour of Colliding Solids, Dover Publications, Mineola, 2001.

[13] J. Lin and L. Qu, "Feature extraction based on morlet wavelet and its application for mechanical fault diagnosis," Journal of Sound and Vibration, vol. 234, no. 1, pp. 135-148, 2000.

[14] Y.-T. Sheen and C. K. Hung, "Constructing a wavelet-based envelope function for vibration signal analysis," Mechanical Systems and Signal Processing, vol. 18, no. 1, pp. 119-126, 2004.

[15] C. C. Liu and Z. Qiu, "A method based on Morlet wavelet for extracting vibration signal envelope," in Proceedings of the 5th International Conference on Signal Processing, pp. 337-340, 2000.

[16] A. Teolis, Computational Signal Processing with Wavelets, Birkhäauser, Boston, Mass, USA, 1st edition, 1998.

[17] L. A. Wong and J. C. Chen, "Nonlinear and chaotic behavior of structural system investigated by wavelet transform techniques," International Journal of Non-Linear Mechanics, vol. 36, no. 2, pp. 221-235, 2001.

[18] S. G. Mallat, A Wavelet Tour of Signal Processing, Academic Press, San Diego, Calif, USA, 2nd edition, 1999.

[19] Q. Meng, M. Zhang, and G. Zhou, "The study on SMA Pseudo-Rubber Metal damper's control efficiency on aseismic pounding in high-pier bridge in shaking table testing," Advanced Materials Research, vol. 163-167, pp. 4429-4441, 2011. 

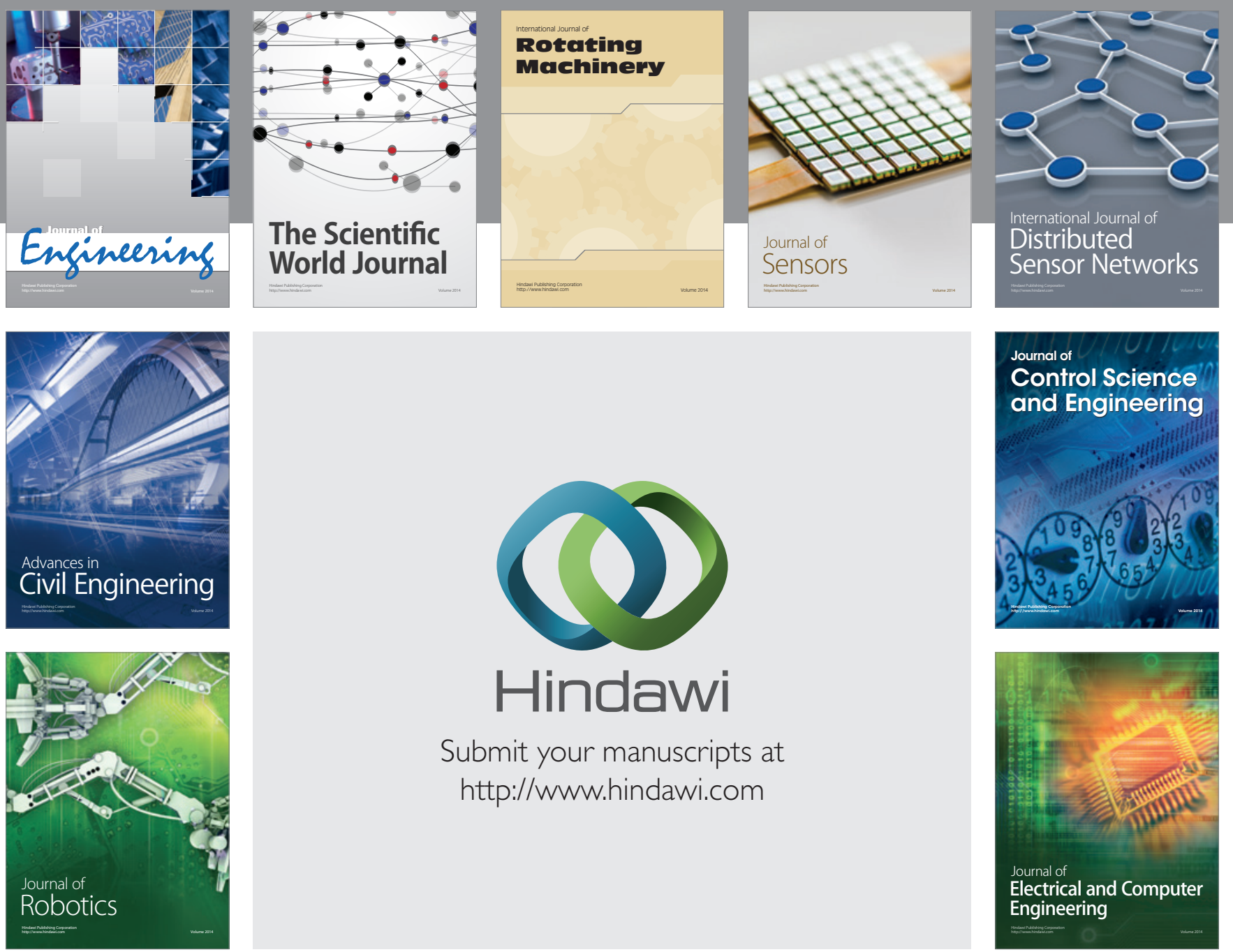

Submit your manuscripts at

http://www.hindawi.com
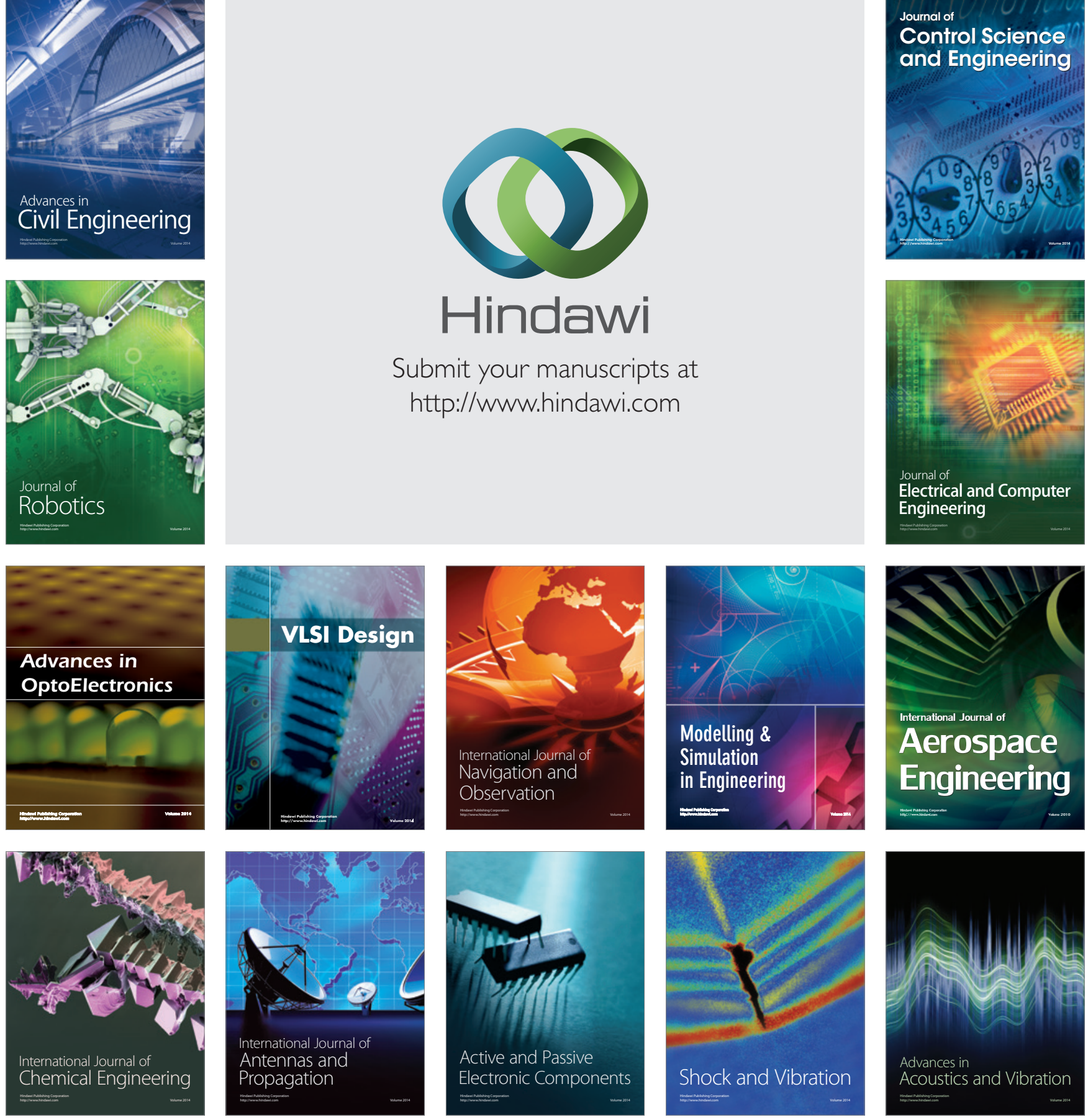